Aim of the study: The incidence of melanoma is increasing rapidly worldwide. Metastatic melanoma is still an incurable disease, although an era of new drugs is approaching. Current methods to predict outcomes in patients with advanced, metastatic melanoma are limited. A retrospective analysis of a contemporary large group of advanced melanomas was performed to determine clinical prognostic factors that accurately predict survival in patients with metastatic melanoma before the era of new targeted/immunological therapy.

Material and methods: The retrospective analysis of 427 patients with metastatic melanoma treated between 1995 and 2005 at two reference oncological centres.

Results: The median overall survival time (OS) was 7.1 months ( $95 \%$ Cl: 6.77.9) and the 1-year, 2-year and 5-year survival rates were $32.3 \% ; 12.5 \% ; 3.9 \%$, respectively. The median progressionfree survival time (PFS) after the first line of treatment was 3.5 months $(95 \% \mathrm{Cl}$ : 3.1-3.8). There were $19.1 \%$ objective responses (CR $-6.1 \%, P R-13.0 \%$ ) and SD $-45.5 \%$ after the first line of therapy. The most common adverse events were anaemia, neutropenia, thrombocytopenia, nausea and vomiting. In multivariate analyses: PS (performance status) 0-1, normal serum levels of lactate dehydrogenase (LDH) and aspartate transaminase (AspAT), older age in women, palliative surgical treatment and palliative radiotherapy, type of the first line of therapy (DTIC), and metastatic melanoma of unknown primary site were independent positive predictors for survival.

Conclusions: The survival rate of patients with metastatic melanoma has not changed significantly over the last years. We identified a set of independent positive predictors for OS treated with systemic therapy. DTIC still may be useful in treatment of patients in a good general condition and with normal serum levels of LDH. Because the results of treatment of metastatic melanoma are still not satisfactory, the majority of patients should be treated within prospective, randomized clinical trials.

Key words: melanoma, metastases, therapy, outcomes, prognostic factors.

\section{Results of systemic treatment of cutaneous melanoma in inoperable stage III and IV}

\author{
Bożena Cybulska-Stopa ${ }^{1}$, Marta Skoczek², Marek Ziobro ${ }^{1}$, Tomasz Świtaj”, \\ Sławomir Falkowski ${ }^{3}$, Tadeusz Morysiński ${ }^{3}$, Marcin Hetnat ${ }^{4}$, Ida Cedrych ${ }^{1}$, \\ Piotr Rutkowski ${ }^{3}$
}

1Department of Systemic and Generalized Malignancies, Centre of Oncology, Maria Skłodowska-Curie Memorial Institute Cracow Branch, Poland 2Department of Chemiotherapy, Szpital Specjalistyczny JP2, Cracow, Poland ${ }^{3}$ Klinika Mięsaków Tkanek Miękkich i Kości oraz Czerniaków, Centre of Oncology, Maria Skłodowska-Curie Memorial Institute, Warsaw, Poland

${ }^{4}$ Department of Radiotherapy, Centre of Oncology, Maria Skłodowska-Curie Memorial Institute Cracow Branch, Poland

\section{Introduction}

Skin melanomas are relatively rare neoplasms; the standardised incidence rate in Poland is about 4/100 000 [1]. However, in recent years there has been a sudden growth in the incidence. It is estimated that in the years 1982-2002 the morbidity increased three-fold [2].

Prognosis in cutaneous melanoma depends strongly on the primary stage according to TNM; thus 5- and 10-year survival rates in stage IA are $97 \%$ and 93\% respectively, while in stage IIIC they are only $53 \%$ and $39 \%$. Stage IV metastasis to distant organs - is characterised by the worst prognosis, and 1 -year survival is $62 \%$ for M1a, $53 \%$ for M1b and 33\% for M1c [3].

Despite advances in diagnosis and treatment, the inoperable stage III and stage IV melanoma remains an incurable disease, and the median survival time in those stages is 6-11 months [4-7]. For this reason numerous patients with inoperable skin melanoma are still being included in clinical trials with various chemotherapy regimens, immunotherapy and targeted therapies, which give hope for changing the results of treatment in the near future. So far the greatest advance in the treatment of those patients has been connected with introduction of monoclonal anti-CTLA-4 antibodies, which have been registered in Europe for the treatment of advanced (unresectable or metastatic) melanoma in adults after previous therapy failure, but they lack predictive factors for response to treatment with simultaneous considerable side effects of this therapy. The second promising treatment is molecular-targeted therapy with tyrosine kinase inhibitors acting on BRAF or KIT protein mutations pathways. Currently there is no consensus regarding the role of systemic treatment in this group of patients, and in the era of novel treatment methods and still numerous group of patients treated only symptomatically, it is vital to define the results and potential role of chemotherapy in treatment of advanced, unresectable skin melanoma. Melanomas are cancers with low chemosensitivity, and the benefit from the chemotherapy is confined to selected subgroups of patients [8-10]. The most important drug used in monotherapy and as part of multidrug regimens is dacarbazine (DTIC), the only chemotherapeutic to have been approved by the FDA for the treatment of melanoma. The objective response rate to treatment with DTIC, after analysing 23 randomised, controlled clinical trials, was found to be $15.3 \%$ [11]. The impact of dacarbazine on overall survival was not evaluated, because no clinical trials comparing dacarbazine with placebo have been conducted [12]. The next group of chemotherapeu- 
tics is vinca alkaloids: vindesine, vinblastine, used mostly with other chemotherapeutics, taxanes (mostly paclitaxel), and nitrosourea derivatives: carmustine (BCNU), lomustine (CCNU) and fotemustine. Their activity in monotherapy is similar to that of dacarbazine, but unfortunately they are characterised by greater toxicity, especially myelosuppression. Although fotemustine is not registered by the FDA, it is available in Europe and used for treating melanoma, especially in the case of brain metastases. Immunotherapy with interferon $\alpha-2 b$ and interleukin 2 plays an important role in the treatment of melanoma. High dose interleukin 2 (HD IL-2) was approved by the FDA in 1998 for the treatment of patients with metastatic melanoma. The basis for registration was achieving durable complete responses correlating with prolonged overall survival in a small group of patients. Because of the high percentage of severe adverse events that can occur during HD IL-2 treatment, such immunotherapy is recommended for carefully selected younger patients, in good general condition [14, 15].

Because of an opinion that a combination of a few chemotherapeutics may increase the rate of objective responses to the treatment and lengthen overall survival, research on multidrug chemotherapy was carried out. However, no expected results were found, and the increase in objective responses to the treatment did not result in overall survival lengthening [16-19]. Similar results were acquired by adding interleukin 2 and/or interferon $\alpha-2 b$ to the multidrug schemes (biochemotherapy). It increased the objective responses to the treatment with a simultaneous considerable increase in treatment toxicity, without a significant impact on overall survival [20-22].

Therefore, research regarding factors that predict the clinical course of advanced melanomas and their response to systemic treatment is being carried out in order to establish optimal criteria for selecting patients for the treatment. It is vital to fully determine the set of prognostic and predictive factors within the group of melanoma patients with unresectable metastases to regional lymph nodes/in-transit metastases or metastases to distant organs treated with classical therapeutic methods, taking into consideration the approaching introduction of drugs with new mechanisms of action which will change the landscape of advanced melanoma therapy.

\section{Material and methods}

We performed a retrospective analysis on 427 patients with inoperable stage III and IV melanoma, aged 51.4 on average (19-82 years), comprising 236 (55.27\%) men and 191 $(44.73 \%)$ women who started systemic treatment in the Department of Systemic and Generalized Malignancies of the Centre of Oncology (Cracow Branch) - 177 (41.45\%) patients and in the Department of Soft Tissue/Bone Sarcoma and Melanoma of the Centre of Oncology (Warsaw) - 250 (58.55\%) patients in the years 1995-2005. The analysed group included only patients with histologically confirmed diagnosis of skin melanoma. Patients with ocular and mucosal melanoma were not included in the analysis [8, 23-25]. Complete information on tested clinical factors, survival and treatment method was gathered. Evaluation of time-varying clin- ical parameters such as stage of the disease (stage III or IV), affected sites, performance status, menopausal status in women and biochemical parameters was performed when diagnosing inoperable metastasis or disqualifying from surgical treatment. For each patient not only basic epidemiological information was recorded, such as age, sex, and the date of histological diagnosis, but also the date of dissemination of the disease (or qualifying changes as inoperable) and date of possible death.

The disease stage was assessed on the basis of the medical reports of clinical and imaging examinations (chest/bone $X$-ray, bone scan, ultrasonographic tests and CT/MR scans). No PET examinations were performed. In single cases additional data were provided by histological and cytological tests of the material obtained during diagnostic procedures (trepano-biopsy, cerebrospinal fluid collection, palliative resections). In the case of metastasis from an unknown primary melanoma, the date of diagnosis was treated as the date of metastasis.

Any form of systemic therapy used by an oncologist which is not standard symptomatic/supportive care was taken as systemic treatment (analgesics, treatment of non-oncological comorbidities, typical symptomatic treatments, e.g. administering dexamethasone in brain oedema, were excluded). Some forms of treatment with questionable efficacy, e.g. hormonal therapy with tamoxifen and medroxyprogesterone, were classified as systemic treatment. This is justified by the fact that, for the time being, there are no data from randomized clinical trials confirming the influence of any form of systemic treatments including dacarbazine (and excluding anti-CTLA4 and BRAF inhibitors) on overall survival in the entire group of patients with metastatic melanoma. The following active drugs were used in monotherapy or in multidrug regimens: dacarbazine (DTIC), carmustine (BCNU), vincristine, vinblastine, cisplatin, carboplatin, cyclophosphamide, dactinomycin, methotrexate, 5-fluorouracil, bleomycin, temozolomide, paclitaxel, tamoxifen, medroxyprogesterone, interleukin-2, interferon $\alpha-2 b$.

The kind of systemic treatment was classified in one of the following categories [15, 17, 18, 41]: DTIC monotherapy, multidrug chemotherapy with DTIC, multidrug chemotherapy without DTIC, immunotherapy, hormonal therapy, or other monotherapy (without DTIC). Single cases of chemoimmunotherapy (chemotherapy + interleukin-2) were classified as multidrug chemotherapy with respect to simultaneously used cytostatics (multidrug chemotherapy with or without DTIC), because it would be necessary to create an additional (small) category of treatment.

Response to treatment was evaluated according to RECIST - Response Evaluation Criteria in Solid Tumors v. 1.0. The assessment of toxicity in all cases was based on CTCAE - Common Terminology Criteria for Adverse Events v. 3.0. In all cases adverse symptoms from respective organs and systems are scored on a four-point scale (1-4). All forms of excision of metastatic lesions in patients with distant metastasis of melanoma were considered as palliative resection. Those were most commonly resections within soft tissues, brain, gastrointestinal tract, and in a few cases resections of metastatic changes from lungs or liver. Radiotherapy of dis- 
tant metastases (mainly in central nervous system and skeletal system) were considered palliative radiotherapy.

\section{Statistical analysis}

Descriptive analysis was performed; averages and standard deviations, and medians and values for numerical variables as well as category rate distribution for categorical variables were given. For comparison of distribution of categorical variables between groups the chi-square test was used, and in smaller groups in four-field tables, Fisher's exact test. The overall survival (OS) was counted from the date of diagnosis of unresectable metastases in stage III/IV to the date of death, and in patients who lived after the end of the test (censored observations) to the date of the last follow-up. The date of starting the first cycle of the next treatment was taken as the starting date to calculate the time to progression after every line of therapy. The date of progression was adopted as the ending date (uncensored observations), or, if not exactly known, the date of introducing the next line of treatment or the date of the patient's death. In censored observations (patients who did not die and had no features of progression during the last visit) the date of the last follow-up is the end date.

The survival curves, the survival time median with confidence interval and 6-month, 1-, 2-, and 3-year survivals were estimated by Kaplan-Meier's method. Because of the low rate of patients who survived and were observed for a longer time, no rate of patients who survived over 3 years was assessed for overall survival estimations, as well as patients who survived over 2 years in case of progression after successive lines of treatment.

The univariate analysis of factors having an influence on overall survival was performed by comparing survival curves with the log-rank test, and in the case of non-parallel curves the $p$ values for Wilcoxon's test were also given (differences in the initial parts of a curve). Independent prognostic factors having an influence on the survival time were searched for with multivariate analysis using the Cox proportional hazards regression model including factors with a $p$-value $\leq 0.1$ in univariate analysis. None of the tested factors diverged substantially from the assumption of parallel curves. Both statistically significant factors and those which changed the other parameters' values were included within the model using the backward selection method. Also statistically significant interactions between the variables were included in the model. Differences were considered statistically significant if $p$-values were $<0.05$. The statistical analysis was performed with SAS v. 9.1 software.

\section{Results}

In the entire analysed group the median overall survival (OS) was 7.1 months (95\% Cl: 6.7-7.9); 1-, 2-, and 5-year OS rates were $32.3 \%, 12.5 \%$, and $3.9 \%$, respectively (Fig. 1).

\section{Univariate analysis for OS}

The results of the univariate analysis for individual variables are shown in Table 1. The variables having a significant impact on OS were: sex, age at the time of diagnosis of unresectable metastases in women, initial activity of lactate dehydrogenase (LDH) (Fig. 2), AlAT and AspAT in serum, initial number of affected sites (organs), metastases in lungs and liver, performance status (Fig. 3), disease stage according to the AJCC/UICC (American Joint Committee on Cancer/International Union Against Cancer) staging system, type of firstline treatment, response to first-line treatment (Fig. 4), num-

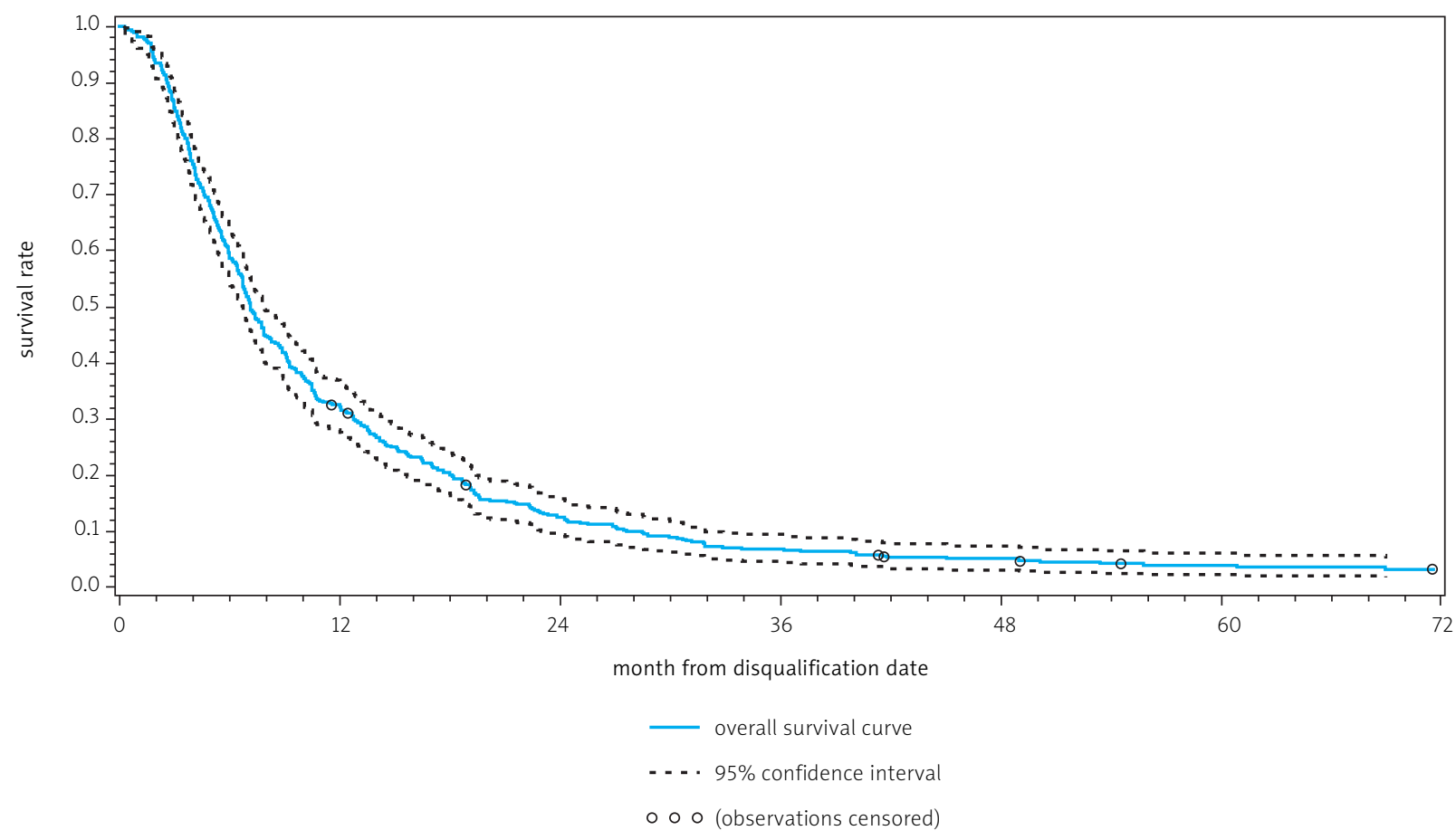

Fig. 1. OS curve for the whole group of patients diagnosed with advanced melanoma $(n=427)$ 
Table 1. Results of single-factor analysis with estimated number of surviving patients depending on variable option

\begin{tabular}{|c|c|c|c|c|c|c|c|c|}
\hline Tested variable & Variable option & $N$ & $\begin{array}{l}\text { Median } \\
\text { in months }\end{array}$ & $\begin{array}{c}95 \% \mathrm{Cl} \\
\text { for median }\end{array}$ & $\begin{array}{c}\text { 1-year } \\
\text { survival [\%] }\end{array}$ & $\begin{array}{c}\text { 2-year } \\
\text { survival [\%] }\end{array}$ & $\begin{array}{c}\text { 5-year } \\
\text { survivals [\% }\end{array}$ & $\begin{array}{l}\text { Pvalue } \\
\text { 6] (significance } \\
\text { of difference for } \\
\text { all survival curves) }\end{array}$ \\
\hline whole group & & 427 & 7.1 & $6.7-7.9$ & 32.3 & 12.5 & 3.9 & \\
\hline center & $\begin{array}{l}\text { Cracow } \\
\text { Warsaw }\end{array}$ & $\begin{array}{l}177 \\
250\end{array}$ & $\begin{array}{l}7.1 \\
7.1\end{array}$ & $\begin{array}{l}6.7-9.0 \\
6.3-8.2\end{array}$ & $\begin{array}{l}30.5 \\
33.6\end{array}$ & $\begin{array}{l}10.7 \\
13.8\end{array}$ & $\begin{array}{l}3.2 \\
4.4\end{array}$ & 0.9702 \\
\hline sex & $\begin{array}{l}\text { female } \\
\text { male }\end{array}$ & $\begin{array}{r}191 \\
236\end{array}$ & $\begin{array}{l}10 \\
6.5\end{array}$ & $\begin{array}{c}7.8-12.7 \\
5.8-7.0\end{array}$ & $\begin{array}{l}44.5 \\
22.5\end{array}$ & $\begin{array}{c}20.0 \\
6.5\end{array}$ & $\begin{array}{l}6.6 \\
1.7\end{array}$ & $<0.0001$ \\
\hline menopause & $\begin{array}{l}\text { yes } \\
\text { no }\end{array}$ & $\begin{array}{r}85 \\
105\end{array}$ & $\begin{array}{l}12.7 \\
7.8\end{array}$ & $\begin{array}{l}8.8-15.1 \\
6.4-10.2\end{array}$ & $\begin{array}{l}51.8 \\
38.1\end{array}$ & $\begin{array}{l}24.7 \\
15.4\end{array}$ & $\begin{array}{l}8.6 \\
4.9\end{array}$ & 0.0812 \\
\hline $\begin{array}{l}\text { age at removing primary } \\
\text { change (in years) }\end{array}$ & $\begin{array}{l}<30 \\
30+\end{array}$ & $\begin{array}{r}35 \\
392\end{array}$ & $\begin{array}{l}6.1 \\
7.1\end{array}$ & $\begin{array}{l}3.4-9.2 \\
6.7-8.0\end{array}$ & $\begin{array}{l}28.6 \\
32.7\end{array}$ & $\begin{array}{c}8.6 \\
12.9\end{array}$ & $\begin{array}{l}\text { ne } \\
4.0\end{array}$ & 0.357 \\
\hline $\begin{array}{l}\text { age at disqualification } \\
\text { from radical treatment or } \\
\text { distant metastasis (in years) }\end{array}$ & $\begin{array}{l}<30 \\
30+\end{array}$ & $\begin{array}{r}34 \\
393\end{array}$ & $\begin{array}{l}5.4 \\
7.1\end{array}$ & $\begin{array}{l}3.3-9.1 \\
6.7-8.0\end{array}$ & $\begin{array}{l}26.5 \\
32.8\end{array}$ & $\begin{array}{c}5.9 \\
13.1\end{array}$ & $\begin{array}{l}\text { ne } \\
4.0\end{array}$ & $\begin{array}{c}0.2049 \text { (log-rank) } \\
0.0357 \\
\text { (Wilcoxon) }\end{array}$ \\
\hline $\begin{array}{l}\text { age at disqualification } \\
\text { from radical treatment } \\
\text { or distant metastasis - } \\
\text { among females (in years) }\end{array}$ & $\begin{array}{l}<30 \\
30+\end{array}$ & $\begin{array}{r}15 \\
176\end{array}$ & $\begin{array}{c}5.7 \\
10.6\end{array}$ & $\begin{array}{l}3.3-7.8 \\
8.2-12.9\end{array}$ & $\begin{array}{c}20 \\
46.6\end{array}$ & $\begin{array}{l}\text { ne } \\
21.7\end{array}$ & $\begin{array}{l}\text { ne } \\
7.1\end{array}$ & 0.0029 \\
\hline $\begin{array}{l}\text { age at disqualification from } \\
\text { radical treatment or distant } \\
\text { metastasis (in years) }\end{array}$ & $\begin{array}{l}<30 \\
30-<50 \\
50+\end{array}$ & $\begin{array}{r}34 \\
159 \\
234\end{array}$ & $\begin{array}{l}5.4 \\
6.4 \\
7.8\end{array}$ & $\begin{array}{l}3.3-9.1 \\
5.6-7.2 \\
7.1-9.6\end{array}$ & $\begin{array}{l}26.5 \\
27.7 \\
36.3\end{array}$ & $\begin{array}{c}5.9 \\
10.7 \\
14.7\end{array}$ & $\begin{array}{l}\text { ne } \\
1.3 \\
5.7\end{array}$ & 0.0589 \\
\hline $\begin{array}{l}\text { age at disqualification from } \\
\text { radical treatment or distant } \\
\text { metastasis-among females } \\
\text { (in years) }\end{array}$ & $\begin{array}{l}<30 \\
30-<50 \\
50+\end{array}$ & $\begin{array}{l}15 \\
78 \\
98\end{array}$ & $\begin{array}{r}5.7 \\
7.6 \\
12.8\end{array}$ & $\begin{array}{c}3.3-7.8 \\
6.0-10.6 \\
10.4-15.6\end{array}$ & $\begin{array}{l}20.0 \\
37.2 \\
54.1\end{array}$ & $\begin{array}{c}\text { ne } \\
13.7 \\
28.1\end{array}$ & $\begin{array}{c}\text { ne } \\
2.7 \\
10.6\end{array}$ & 0.0008 \\
\hline $\begin{array}{l}\text { time from diagnosis to } \\
\text { metastasis (in months) }\end{array}$ & $\begin{array}{l}<36 \\
\geq 36\end{array}$ & $\begin{array}{r}339 \\
88\end{array}$ & $\begin{array}{l}6.8 \\
8.4\end{array}$ & $\begin{array}{l}6.3-7.8 \\
7.2-11.3\end{array}$ & $\begin{array}{l}30.4 \\
39.8\end{array}$ & $\begin{array}{l}10.9 \\
18.8\end{array}$ & $\begin{array}{l}3.9 \\
3.9\end{array}$ & $\begin{array}{c}0.1289 \\
(\text { log-rank) } \\
0.0490 \text { (Wilcoxon) }\end{array}$ \\
\hline $\begin{array}{l}\text { disease duration before } \\
\text { diagnosis of melanoma } \\
\text { metastasis }\end{array}$ & $\begin{array}{l}\text { after primary } \\
\text { source excision } \\
\text { after regional lymph } \\
\text { node metastasis } \\
\text { metastasis during } \\
\text { diagnosis }\end{array}$ & $\begin{array}{r}124 \\
277 \\
26\end{array}$ & $\begin{array}{r}6.8 \\
7.1 \\
12.4\end{array}$ & $\begin{array}{l}5.7-9.3 \\
6.5-7.8 \\
7.5-19.1\end{array}$ & $\begin{array}{l}31.5 \\
31.0 \\
50.0\end{array}$ & $\begin{array}{l}15.3 \\
10.4 \\
21.6\end{array}$ & $\begin{array}{l}3.0 \\
3.7 \\
\text { ne }\end{array}$ & 0.114 \\
\hline $\begin{array}{l}\text { metastasis during } \\
\text { diagnosis }\end{array}$ & $\begin{array}{l}\text { no } \\
\text { yes }\end{array}$ & $\begin{array}{r}401 \\
26\end{array}$ & $\begin{array}{c}7 \\
12.4\end{array}$ & $\begin{array}{l}6.5-7.8 \\
7.5-19.1\end{array}$ & $\begin{array}{l}31.2 \\
50.0\end{array}$ & $\begin{array}{l}11.9 \\
21.6\end{array}$ & $\begin{array}{l}3.6 \\
\text { ne }\end{array}$ & 0.0599 \\
\hline $\begin{array}{l}\text { metastasis from } \\
\text { unknown primary source }\end{array}$ & $\begin{array}{l}\text { no } \\
\text { yes }\end{array}$ & $\begin{array}{r}398 \\
29\end{array}$ & $\begin{array}{c}7 \\
10.8\end{array}$ & $\begin{array}{l}6.4-7.8 \\
7.1-18.8\end{array}$ & $\begin{array}{l}31.4 \\
44.8\end{array}$ & $\begin{array}{l}12.0 \\
19.4\end{array}$ & $\begin{array}{l}3.6 \\
\text { ne }\end{array}$ & 0.0935 \\
\hline ECOG & $\begin{array}{l}0 \\
1 \\
2 \\
3\end{array}$ & $\begin{array}{r}194 \\
177 \\
49 \\
6\end{array}$ & $\begin{array}{c}9 \\
6.7 \\
4.6 \\
1.8\end{array}$ & $\begin{array}{c}7.8-10.0 \\
5.6-7.8 \\
2.9-6.9 \\
1.4-4.3\end{array}$ & $\begin{array}{c}38.1 \\
31.1 \\
16.3 \\
\text { ne }\end{array}$ & $\begin{array}{c}17.2 \\
8.6 \\
9.7 \\
\text { ne }\end{array}$ & $\begin{array}{l}4.9 \\
2.7 \\
\text { ne } \\
\text { ne }\end{array}$ & $<0.0001$ \\
\hline $\begin{array}{l}\text { baseline LDH activity } \\
\text { in serum }\end{array}$ & $\begin{array}{l}\text { normal } \\
\text { elevated }\end{array}$ & $\begin{array}{l}191 \\
145\end{array}$ & $\begin{array}{l}9.6 \\
5.2\end{array}$ & $\begin{array}{l}7.6-11.5 \\
4.2-6.4\end{array}$ & $\begin{array}{l}42.9 \\
19.3\end{array}$ & $\begin{array}{c}17.9 \\
5.7\end{array}$ & $\begin{array}{l}6.4 \\
1.4\end{array}$ & $<0.0001$ \\
\hline $\begin{array}{l}\text { baseline AIAT activity } \\
\text { in serum }\end{array}$ & $\begin{array}{l}\text { normal } \\
\text { elevated }\end{array}$ & $\begin{array}{r}289 \\
64\end{array}$ & $\begin{array}{l}7.4 \\
5.6\end{array}$ & $\begin{array}{l}6.7-9.1 \\
4.3-7.2\end{array}$ & $\begin{array}{l}34.6 \\
21.9\end{array}$ & $\begin{array}{c}13.3 \\
7.8\end{array}$ & $\begin{array}{l}5.1 \\
\text { ne }\end{array}$ & 0.002 \\
\hline $\begin{array}{l}\text { baseline AspAT activity } \\
\text { in serum }\end{array}$ & $\begin{array}{l}\text { normal } \\
\text { elevated }\end{array}$ & $\begin{array}{r}301 \\
53\end{array}$ & $\begin{array}{l}7.5 \\
5.1\end{array}$ & $\begin{array}{l}6.8-9.1 \\
3.7-7.0\end{array}$ & $\begin{array}{l}35.5 \\
13.2\end{array}$ & $\begin{array}{c}13.8 \\
3.8\end{array}$ & $\begin{array}{l}4.9 \\
\text { ne }\end{array}$ & $<0.0001$ \\
\hline baseline HBG level & $\begin{array}{l}\text { normal } \\
\text { reduced }\end{array}$ & $\begin{array}{r}312 \\
68\end{array}$ & $\begin{array}{l}7.3 \\
6.5\end{array}$ & $\begin{array}{l}6.7-9.0 \\
5.7-8.5\end{array}$ & $\begin{array}{l}36.5 \\
20.6\end{array}$ & $\begin{array}{c}13.9 \\
5.9\end{array}$ & $\begin{array}{l}4.8 \\
2.9\end{array}$ & 0.1557 \\
\hline $\begin{array}{l}\text { number of involved loca- } \\
\text { tions (organs) at the time } \\
\text { of disqualification from }\end{array}$ & $\begin{array}{l}1 \\
2 \\
3 \text { or }>3\end{array}$ & $\begin{array}{r}172 \\
213 \\
38\end{array}$ & $\begin{array}{l}7.9 \\
6.7 \\
8.6\end{array}$ & $\begin{array}{l}7.0-9.6 \\
5.5-7.3 \\
5.6-11.3\end{array}$ & $\begin{array}{l}34.9 \\
30.0 \\
34.2\end{array}$ & $\begin{array}{c}15.7 \\
9.5 \\
15.8\end{array}$ & $\begin{array}{l}6.1 \\
\text { ne } \\
2.6\end{array}$ & 0.0335 \\
\hline
\end{tabular}

radical treatment 
Table 1. cont.

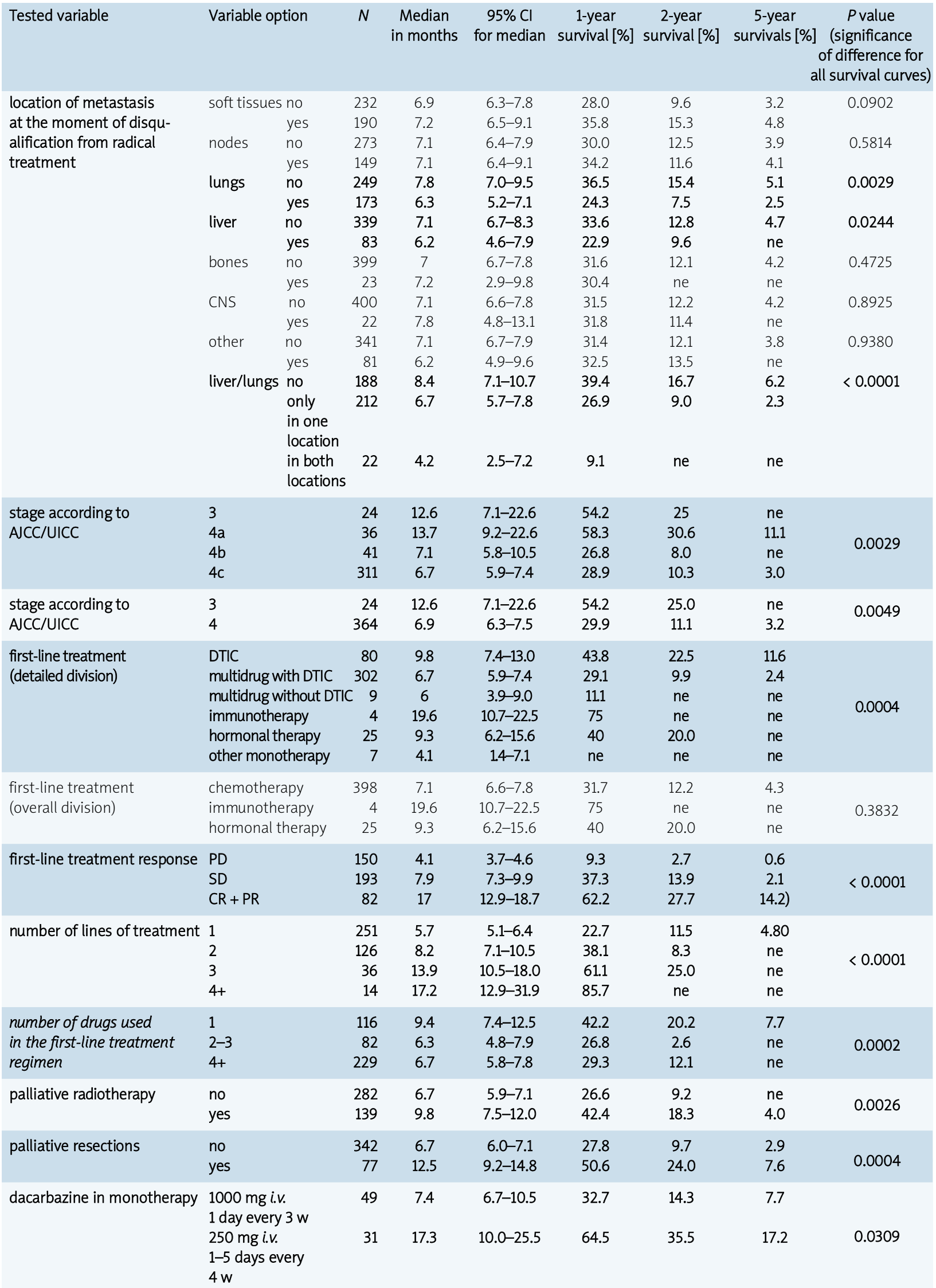




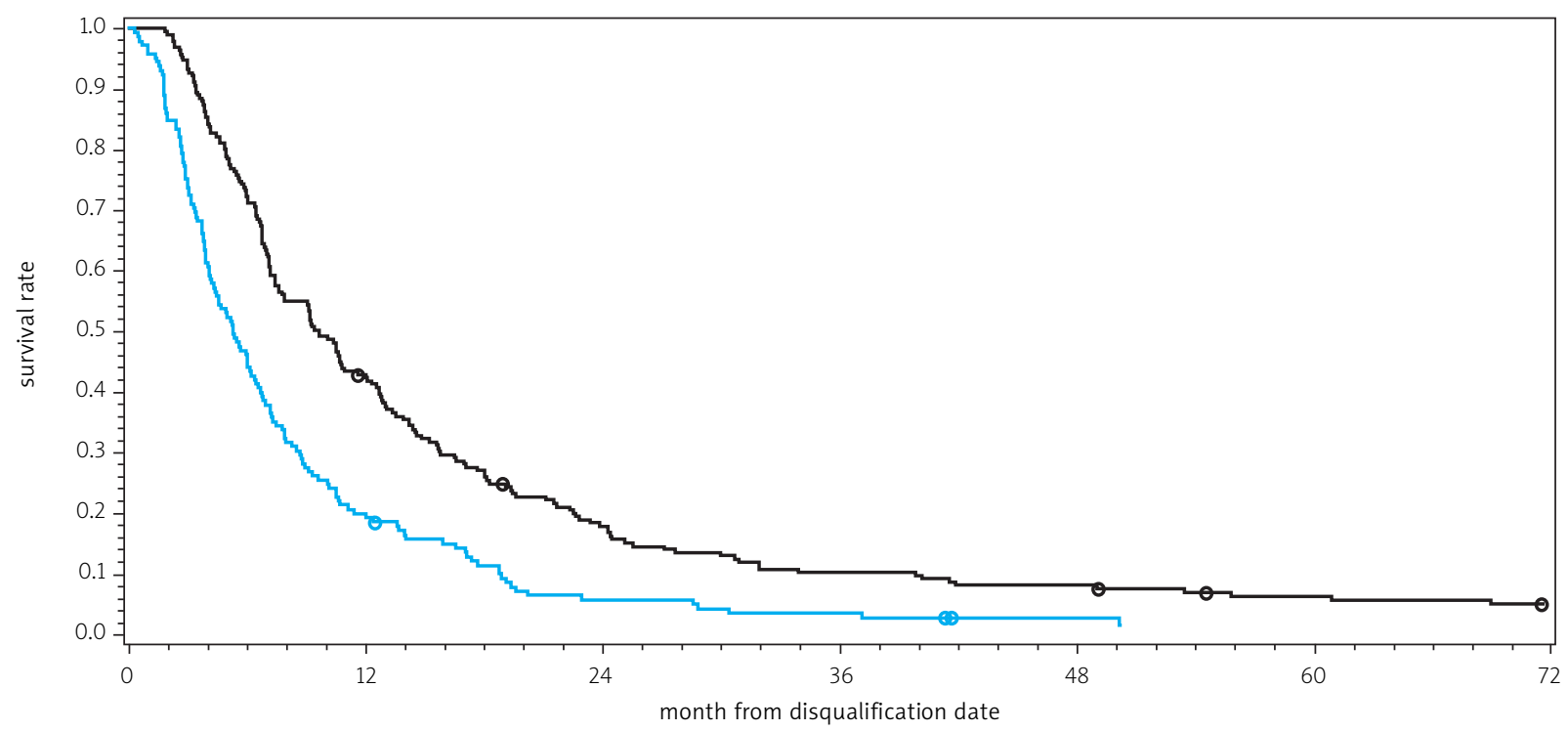

LDH level: — normal

- elevated

○०० observations censored

000 observations censored

Fig. 2. OS curves in relation to baseline LDH activity in serum $(p<0.0001)$

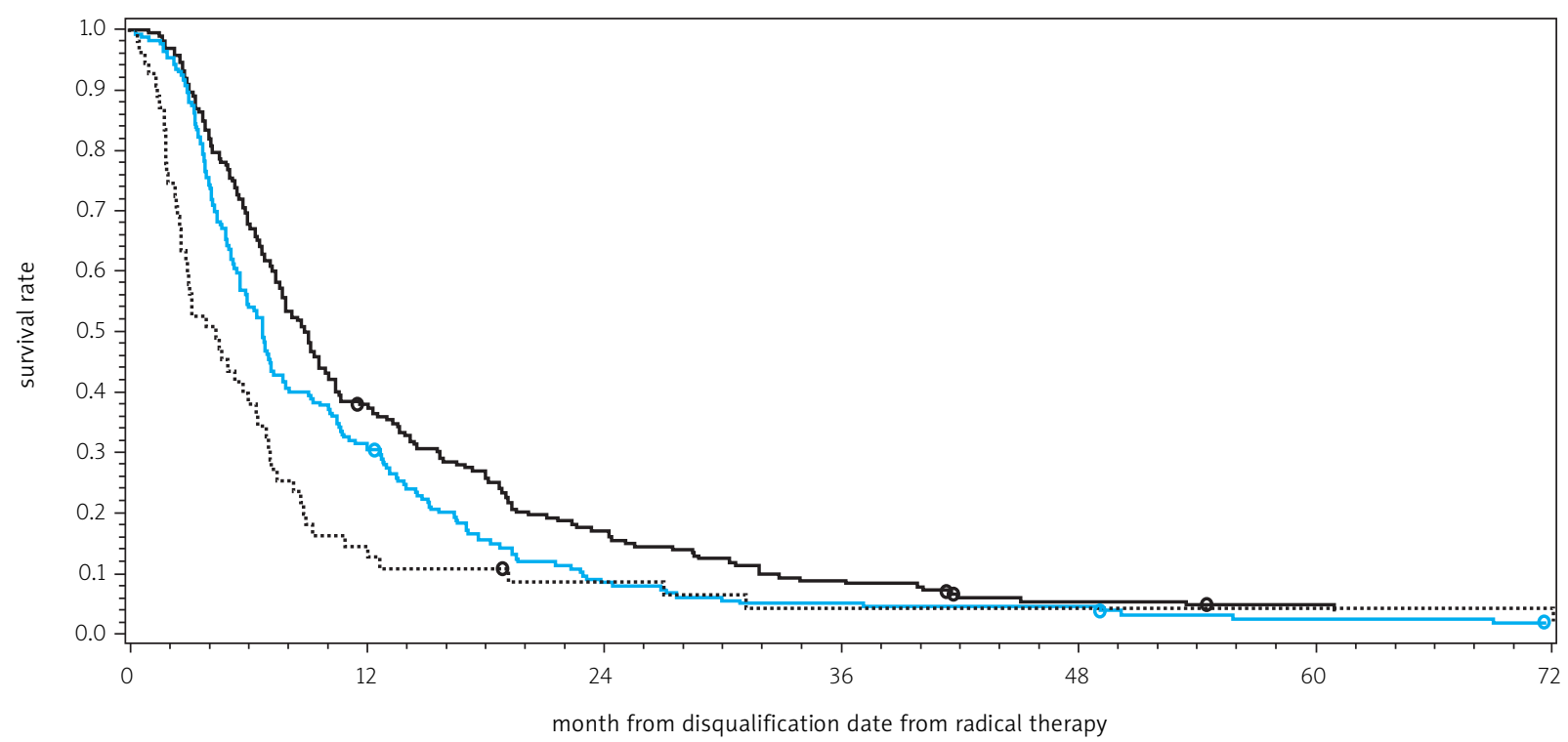

Overall condition according to ECOG: $\overline{000} 0$ (observations censored)

$\overline{000} 1$ (observations censored)

0002 or more (observations censored)

Fig. 3. OS curves depending on patient's general condition $(\mathrm{ECOG})(p<0.0001)$ 


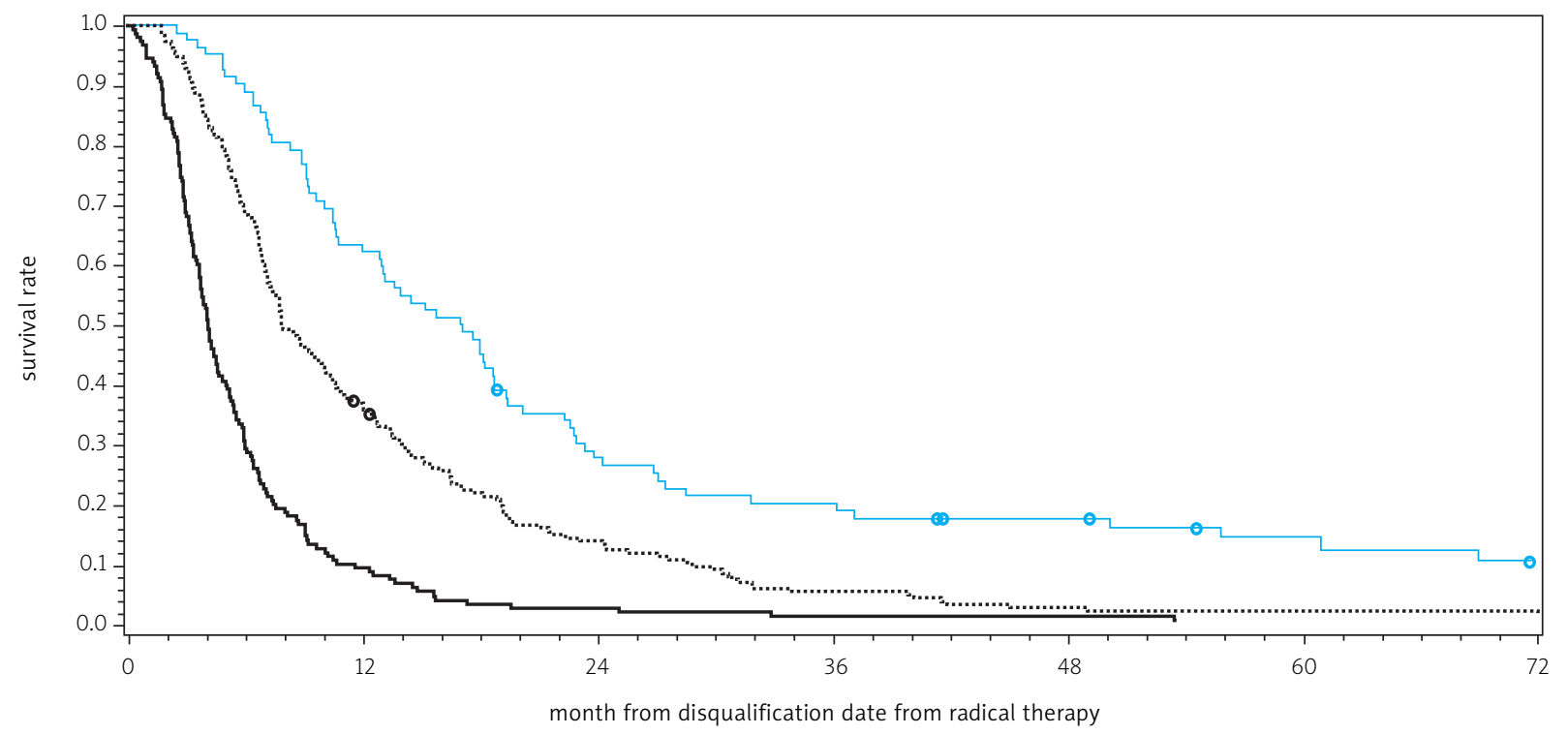

Response to first-line chemotherapy: $\overline{000}$ PD (observations censored)

$\overline{000} \mathrm{CP}+\mathrm{PR}$ (observations censored)

.0. SD (observations censored)

Fig. 4. OS curve in relation to first-line treatment response $(p<0.0001)$

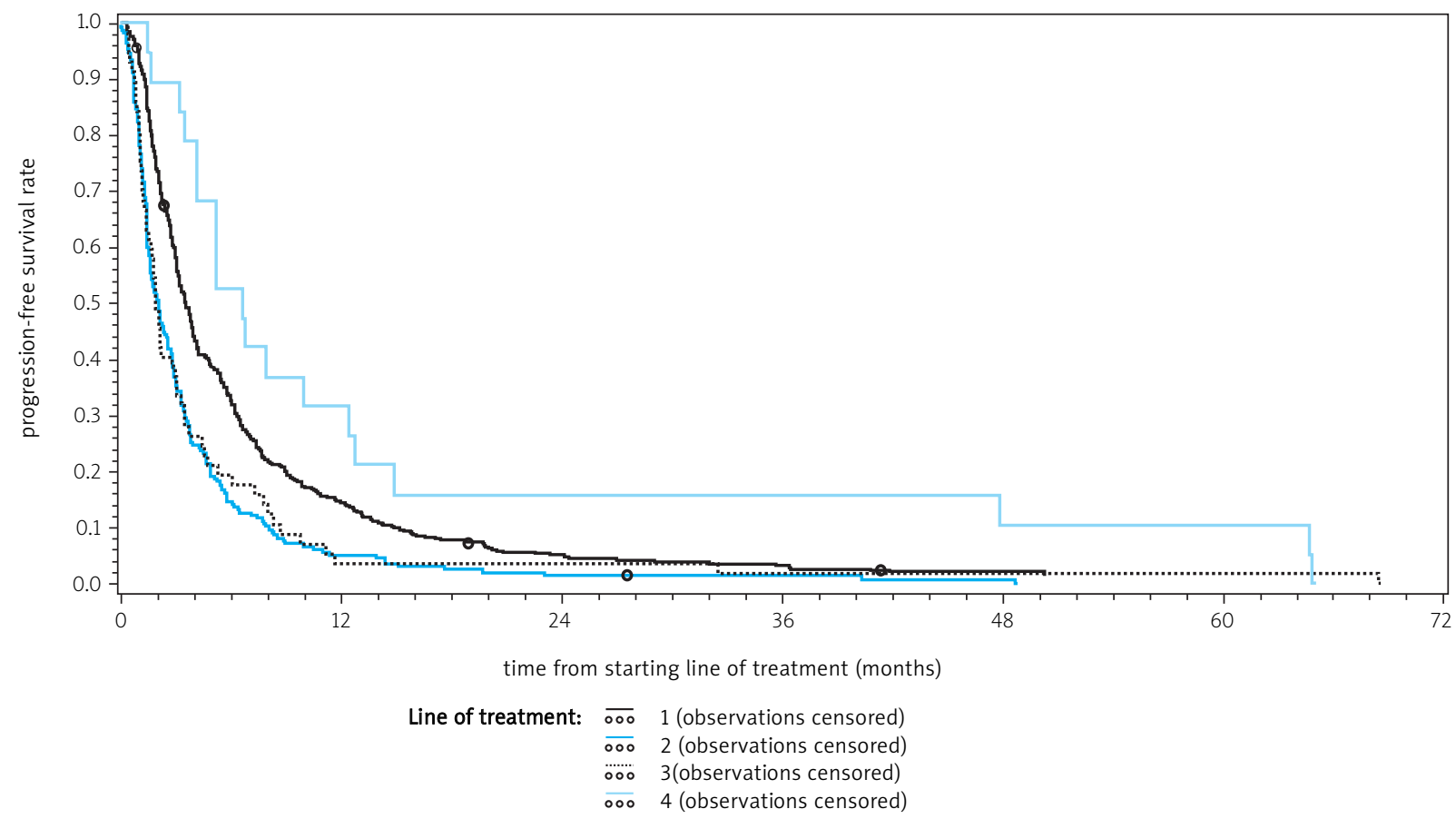

Fig. 5. Curves depicting PFS after successive lines of treatment 
Table 2. Responses to treatment in successive lines

\begin{tabular}{|c|c|c|c|c|c|c|}
\hline Type of treatment & $\begin{array}{c}C R \\
N(\%)\end{array}$ & $\begin{array}{c}\text { PR } \\
N(\%)\end{array}$ & $\begin{array}{c}S D^{*} \\
N(\%)\end{array}$ & $\begin{array}{c}P D \\
N(\%)\end{array}$ & $\begin{array}{c}\text { No data } \\
N\end{array}$ & $\begin{array}{c}\text { All } \\
N\end{array}$ \\
\hline \multicolumn{7}{|c|}{ Responses to first-line treatment depending on therapy type } \\
\hline all & $26(6.1)$ & $55(13.0)$ & $193(45.5)$ & $150(35.4)$ & 3 & 427 \\
\hline DTIC chemotherapy & $8(10.0)$ & $8(10.0)$ & $34(42.5)$ & $30(37.5)$ & 0 & 80 \\
\hline multidrug chemotherapy with DTIC & $18(6.0)$ & $41(13.6)$ & $143(47.5)$ & 99 (32.9) & 1 & 302 \\
\hline multidrug chemotherapy without DTIC & 0 & $1(11.1)$ & $1(11.1)$ & $7(77.8)$ & 0 & 9 \\
\hline immunotherapy & 0 & 0 & $2(66.7)$ & $1(33.3)$ & 1 & 4 \\
\hline hormonal therapy & 0 & $3(12.0)$ & $12(48.0)$ & $10(40.0)$ & 0 & 25 \\
\hline other monotherapy & 0 & $2(33.3)$ & $1(16.7)$ & $3(50.0)$ & 1 & 7 \\
\hline \multicolumn{7}{|c|}{ Responses to second-line treatment depending on therapy type } \\
\hline all & $2(1.0)$ & $11(5.6)$ & $42(21.2)$ & $141(72.1)$ & 6 & 202 \\
\hline DTIC chemotherapy & 0 & $1(11.1)$ & $1(11.1)$ & $7(77.8)$ & 0 & 9 \\
\hline multidrug chemotherapy with DTIC & 0 & $6(7.3)$ & $16(19.5)$ & $60(73.2)$ & 0 & 82 \\
\hline multidrug chemotherapy without DTIC & $1(6.3)$ & 0 & $8(50.0)$ & $6(43.8)$ & 1 & 16 \\
\hline immunotherapy & 0 & 0 & $2(50.0)$ & $2(50.0)$ & 1 & 5 \\
\hline hormonal therapy & $1(1.5)$ & $3(4.5)$ & $14(21.2)$ & $47(72.7)$ & 3 & 69 \\
\hline other monotherapy & 0 & $1(5.0)$ & $1(5.0)$ & $18(90.0)$ & 1 & 21 \\
\hline \multicolumn{7}{|c|}{ Responses to third-line treatment depending on therapy type } \\
\hline all & $1(1.85)$ & $2(3.7)$ & $16(29.64)$ & $35(64.81)$ & 6 & 60 \\
\hline DTIC chemotherapy & 0 & 0 & $1(33.33)$ & $2(66.67)$ & 0 & 3 \\
\hline multidrug chemotherapy with DTIC & $1(4.55)$ & $1(4.55)$ & $8(71.65)$ & $12(54.55)$ & 2 & 24 \\
\hline multidrug chemotherapy without DTIC & 0 & 0 & $6(42.86)$ & $8(57.14)$ & 1 & 15 \\
\hline immunotherapy & 0 & 0 & $1(33.33)$ & $2(66.67)$ & 1 & 4 \\
\hline hormonal therapy & 0 & 0 & 0 & $5(100.0)$ & 1 & 6 \\
\hline other monotherapy & 0 & $1(14.3)$ & 0 & $6(85.71)$ & 1 & 8 \\
\hline \multicolumn{7}{|c|}{ Responses to fourth-line treatment depending on therapy type } \\
\hline all & 0 & $2(11.0)$ & $6(33.3)$ & $10(55.6)$ & 2 & 20 \\
\hline DTIC chemotherapy & 0 & 0 & 0 & 0 & 0 & 0 \\
\hline multidrug chemotherapy with DTIC & 0 & $1(20.0)$ & $2(40.0)$ & $2(40.0)$ & 0 & 5 \\
\hline multidrug chemotherapy without DTIC & 0 & 0 & $1(25.0)$ & $3(75.0)$ & 0 & 4 \\
\hline immunotherapy & 0 & 0 & $2(100.0)$ & 0 & 0 & 2 \\
\hline hormonal therapy & 0 & $1(50.0)$ & 0 & $1(50.0)$ & 2 & 4 \\
\hline other monotherapy & 0 & 0 & $1(20.0)$ & $4(80.0)$ & 0 & 5 \\
\hline
\end{tabular}

ber of lines of treatment, number of used drugs in the firstline treatment regimen, use of palliative radiotherapy and palliative resections.

\section{Analysis of the applied systemic treatment}

Because of the widely discussed impact of systemic treatment on OS, and no definite answer on the effectiveness of the applied therapy in treating metastatic melanomas, a detailed analysis of the applied treatment (Table 2) and its influence on OS was performed. We assessed the possible impact of the number of treatment lines, the number of drugs in the first-line regimen and the kind of therapy used in firstline treatment. Longer survival of patients who received more lines of treatment may be related to survival bias, which means that the person had to live until the next lines, and so by assumption lived longer. However, it is worth noting that in the majority of patients the treatment was changed after 2-3 cycles due to progression or complications, which also influenced the relationship between OS and number of treatment lines. The multidrug therapies containing dacarbazine were used the most often, both the first and the second line of treatment. The less numerous group of patients was treated with dacarbazine in monotherapy or with hormonal therapy. It can be concluded from the analysis that the best therapeutic choice in the first line of treatment within the tested group was dacarbazine in monotherapy.

Also the influence of the responses to the first-line treatment on OS was assessed. A statistically significant influence of the responses to the treatment on OS was demonstrated.

We also found that the rates of objective responses (CR + PR) drop, and they equal $19.1 \%$ in the first line, $6.6 \%$ in the second line, and $5.6 \%$ in the third line. 
Table 3. Influence of clinical factors on progression-free survival after first line of treatment (in months)

\begin{tabular}{|c|c|c|c|c|c|c|c|}
\hline Factor & $\begin{array}{l}\text { Variable } \\
\text { option }\end{array}$ & $N$ & $\begin{array}{c}N \\
\text { progression }\end{array}$ & Average & Median & $\begin{array}{c}95 \% \mathrm{Cl} \\
\text { for median }\end{array}$ & $p$ value \\
\hline whole group & & 427 & 417 & 6.9 & 3.5 & $3.1-3.8$ & \\
\hline type of first-line therapy & $\begin{array}{l}\text { DTIC } \\
\text { multidrug with DTIC } \\
\text { multidrug without DTIC } \\
\text { immunotherapy } \\
\text { hormonal therapy } \\
\text { other monotherapy }\end{array}$ & $\begin{array}{c}80 \\
302 \\
9 \\
4 \\
25 \\
7\end{array}$ & $\begin{array}{c}75 \\
297 \\
9 \\
4 \\
25 \\
7\end{array}$ & $\begin{array}{c}10.1 \\
6.2 \\
3 \\
7.8 \\
9.5 \\
2.6\end{array}$ & $\begin{array}{l}5.5 \\
3.2 \\
1.7 \\
7.2 \\
5.8 \\
2.9\end{array}$ & $\begin{array}{l}3.8-6.4 \\
3.0-3.7 \\
1.5-2.0 \\
3.1-13.6 \\
3.0-9.2 \\
1.4-4.0\end{array}$ & 0.0007 \\
\hline center & $\begin{array}{l}\text { Cracow } \\
\text { Warsaw }\end{array}$ & $\begin{array}{l}177 \\
250\end{array}$ & $\begin{array}{l}175 \\
242\end{array}$ & $\begin{array}{l}6.5 \\
7.1\end{array}$ & $\begin{array}{l}3.5 \\
3.5\end{array}$ & $\begin{array}{l}3.1-4.1 \\
3.0-3.9\end{array}$ & 0.73 \\
\hline sex & $\begin{array}{l}\text { female } \\
\text { male }\end{array}$ & $\begin{array}{l}191 \\
236\end{array}$ & $\begin{array}{l}183 \\
234\end{array}$ & $\begin{array}{c}8 \\
5.7\end{array}$ & $\begin{array}{l}3.8 \\
3.2\end{array}$ & $\begin{array}{l}3.5-5.6 \\
3.0-3.8\end{array}$ & 0.0012 \\
\hline $\begin{array}{l}\text { disqualification age } \\
\text { (in years) }\end{array}$ & $\begin{array}{l}<30 \\
30+\end{array}$ & $\begin{array}{c}34 \\
393\end{array}$ & $\begin{array}{c}33 \\
384\end{array}$ & $\begin{array}{c}4.5 \\
7\end{array}$ & $\begin{array}{l}2.5 \\
3.7\end{array}$ & $\begin{array}{l}1.8-3.9 \\
3.2-4.0\end{array}$ & 0.1392 \\
\hline $\begin{array}{l}\text { state at melanoma } \\
\text { diagnosis }\end{array}$ & $\begin{array}{l}\text { no metastasis } \\
\text { metastasis }\end{array}$ & $\begin{array}{l}401 \\
26\end{array}$ & $\begin{array}{c}392 \\
25\end{array}$ & $\begin{array}{l}6.8 \\
7.1\end{array}$ & $\begin{array}{l}3.5 \\
5.3\end{array}$ & $\begin{array}{l}3.1-3.8 \\
3.9-7.4\end{array}$ & 0.3279 \\
\hline $\begin{array}{l}\text { LDH level at } \\
\text { disqualification }\end{array}$ & $\begin{array}{l}\text { normal } \\
\text { elevated }\end{array}$ & $\begin{array}{l}191 \\
145\end{array}$ & $\begin{array}{l}184 \\
144\end{array}$ & $\begin{array}{l}8.2 \\
5.1\end{array}$ & $\begin{array}{l}3.9 \\
3.1\end{array}$ & $\begin{array}{l}3.4-5.2 \\
2.5-3.8\end{array}$ & 0.0005 \\
\hline $\begin{array}{l}\text { AspAT level at disqualifica- } \\
\text { tion from radical therapy }\end{array}$ & $\begin{array}{l}\text { normal } \\
\text { elevated }\end{array}$ & $\begin{array}{c}301 \\
53\end{array}$ & $\begin{array}{c}293 \\
53\end{array}$ & $\begin{array}{l}7.2 \\
4.5\end{array}$ & $\begin{array}{l}3.7 \\
2.9\end{array}$ & $\begin{array}{l}3.2-4.1 \\
1.9-3.8\end{array}$ & 0.0116 \\
\hline $\begin{array}{l}\text { AlAT level at disqualifica- } \\
\text { tion from radical therapy }\end{array}$ & $\begin{array}{l}\text { normal } \\
\text { elevated }\end{array}$ & $\begin{array}{c}289 \\
64\end{array}$ & $\begin{array}{c}281 \\
64\end{array}$ & $\begin{array}{l}7.3 \\
4.7\end{array}$ & $\begin{array}{c}3.7 \\
3\end{array}$ & $\begin{array}{l}3.2-4.1 \\
2.1-3.9\end{array}$ & 0.0271 \\
\hline $\begin{array}{l}\text { haemoglobin level from } \\
\text { radical therapy initial }\end{array}$ & $\begin{array}{l}\text { normal } \\
\text { reduced }\end{array}$ & $\begin{array}{l}312 \\
68\end{array}$ & $\begin{array}{c}303 \\
67\end{array}$ & $\begin{array}{l}7.2 \\
5.9\end{array}$ & $\begin{array}{l}3.7 \\
3.1\end{array}$ & $\begin{array}{l}3.2-4.0 \\
2.7-4.2\end{array}$ & 0.2633 \\
\hline lung metastasis & $\begin{array}{l}\text { no } \\
\text { yes }\end{array}$ & $\begin{array}{l}249 \\
173\end{array}$ & $\begin{array}{l}242 \\
170\end{array}$ & $\begin{array}{l}6.9 \\
6.2\end{array}$ & $\begin{array}{c}3.8 \\
3\end{array}$ & $\begin{array}{l}3.4-4.8 \\
2.6-3.7\end{array}$ & 0.0808 \\
\hline liver metastasis & $\begin{array}{l}\text { no } \\
\text { yes }\end{array}$ & $\begin{array}{c}339 \\
83\end{array}$ & $\begin{array}{c}329 \\
83\end{array}$ & $\begin{array}{l}7.2 \\
4.8\end{array}$ & $\begin{array}{l}3.5 \\
3.2\end{array}$ & $\begin{array}{l}3.1-3.9 \\
2.6-4.0\end{array}$ & 0.0296 \\
\hline soft tissue metastasis & $\begin{array}{l}\text { no } \\
\text { yes }\end{array}$ & $\begin{array}{l}232 \\
190\end{array}$ & $\begin{array}{l}228 \\
184\end{array}$ & $\begin{array}{l}6.2 \\
7.2\end{array}$ & $\begin{array}{l}3.2 \\
3.8\end{array}$ & $\begin{array}{l}3.0-3.8 \\
3.2-4.6\end{array}$ & 0.1129 \\
\hline lymph nodes metastasis & $\begin{array}{l}\text { no } \\
\text { yes }\end{array}$ & $\begin{array}{l}273 \\
149\end{array}$ & $\begin{array}{l}265 \\
147\end{array}$ & $\begin{array}{l}6.9 \\
6.2\end{array}$ & $\begin{array}{l}3.5 \\
3.7\end{array}$ & $\begin{array}{l}3.0-3.8 \\
3.1-4.7\end{array}$ & 0.9663 \\
\hline CNS metastasis & $\begin{array}{l}\text { no } \\
\text { yes }\end{array}$ & $\begin{array}{c}400 \\
22\end{array}$ & $\begin{array}{c}391 \\
21\end{array}$ & $\begin{array}{l}6.6 \\
9.1\end{array}$ & $\begin{array}{l}3.5 \\
4.4\end{array}$ & $\begin{array}{l}3.0-3.8 \\
3.2-9.0\end{array}$ & 0.1646 \\
\hline bone metastasis & $\begin{array}{l}\text { no } \\
\text { yes }\end{array}$ & $\begin{array}{c}399 \\
23\end{array}$ & $\begin{array}{c}389 \\
23\end{array}$ & $\begin{array}{l}6.8 \\
5.6\end{array}$ & $\begin{array}{l}3.7 \\
2.3\end{array}$ & $\begin{array}{l}3.2-3.9 \\
1.8-3.0\end{array}$ & 0.2506 \\
\hline other metastasis & $\begin{array}{l}\text { no } \\
\text { yes }\end{array}$ & $\begin{array}{c}382 \\
40\end{array}$ & $\begin{array}{c}372 \\
40\end{array}$ & $\begin{array}{c}6.8 \\
6\end{array}$ & $\begin{array}{l}3.5 \\
3.6\end{array}$ & $\begin{array}{l}3.1-3.9 \\
2.2-5.5\end{array}$ & 0.8115 \\
\hline $\begin{array}{l}\text { patient performance } \\
\text { status according to } \\
\text { ECOG scale }\end{array}$ & $\begin{array}{l}0 \\
1 \\
2 \\
3\end{array}$ & $\begin{array}{c}194 \\
177 \\
49 \\
6\end{array}$ & $\begin{array}{c}187 \\
175 \\
48 \\
6\end{array}$ & $\begin{array}{l}7.9 \\
6.5 \\
4.1 \\
2.7\end{array}$ & $\begin{array}{l}3.8 \\
3.5 \\
2.2 \\
1.6\end{array}$ & $\begin{array}{l}3.3-5.4 \\
3.1-4.1 \\
1.8-3.2 \\
1.4-3.5\end{array}$ & 0.0006 \\
\hline $\begin{array}{l}\text { stage according } \\
\text { to AJCC/UICC staging }\end{array}$ & $\begin{array}{l}3 \\
4 a \\
4 b \\
4 c\end{array}$ & $\begin{array}{c}24 \\
12 \\
41 \\
311\end{array}$ & $\begin{array}{c}20 \\
12 \\
41 \\
307\end{array}$ & $\begin{array}{l}11.7 \\
9.1 \\
7.6 \\
5.7\end{array}$ & $\begin{array}{l}6.6 \\
6.6 \\
3.7 \\
3.3\end{array}$ & $\begin{array}{c}3.5-11.2 \\
2.1-13.0 \\
2.4-5.5 \\
3.0-3.7\end{array}$ & 0.0076 \\
\hline
\end{tabular}

Univariate analysis for progression-free survival

The median progression-free survival (PFS) after the first line of treatment was 3.5 months ( $95 \% \mathrm{Cl}$ : 3.1-3.8) for the whole group of patients, and the 6-month PFS rate was $31.8 \%$. We identified the following factors significantly influencing
PFS during the first-line treatment: type of therapy (Fig. 5), sex, initial level of LDH, AspAT, AIAT in serum, metastases to the liver, patient's performance status according to the ECOG scale and staging according to AJCC/UICC (Table 3). The PFS medians after the $1^{\text {st }}, 2^{\text {nd }}$, and $3^{\text {rd }}$ line of systemic treatment 
Table 4. Multi-factor analysis of results for the whole group

\begin{tabular}{|c|c|c|c|c|c|c|}
\hline Factor & & Chi-Square & $\operatorname{Pr}>\chi^{2}$ & $\begin{array}{l}\text { Hazard } \\
\text { ratio }\end{array}$ & $\begin{array}{l}95 \% \\
\text { confid }\end{array}$ & $\begin{array}{l}\text { ratio } \\
\text { imits }\end{array}$ \\
\hline $\begin{array}{l}\text { age of disqualification from } \\
\text { radical therapy among females }\end{array}$ & $\begin{array}{l}30-50 \text { years vs. }<30 \text { years } \\
\geq 50 \text { years vs. }<30 \text { years }\end{array}$ & $\begin{array}{c}0.1916 \\
17.8753\end{array}$ & $\begin{array}{l}0.6616 \\
<0.0001\end{array}$ & $\begin{array}{c}1.60 \\
0.576\end{array}$ & $\begin{array}{l}0.817 \\
0.446\end{array}$ & $\begin{array}{l}1.376 \\
0.744\end{array}$ \\
\hline $\begin{array}{l}\text { age of disqualification from } \\
\text { radical therapy among males }\end{array}$ & $\begin{array}{l}30-50 \text { years vs. }<30 \text { years } \\
\geq 50 \text { years vs. }<30 \text { years }\end{array}$ & $\begin{array}{c}2.3173 \\
1.352\end{array}$ & $\begin{array}{l}0.1279 \\
0.1755\end{array}$ & $\begin{array}{l}1.220 \\
1.178\end{array}$ & $\begin{array}{l}0.944 \\
0.929\end{array}$ & $\begin{array}{l}1.575 \\
1.494\end{array}$ \\
\hline LDH level & elevated vs. N & 16.8556 & $<0.0001$ & 1.697 & 1.318 & 2.184 \\
\hline AspAT level & elevated vs. N & 9.737 & 0.0023 & 1.709 & 1.210 & 2.413 \\
\hline ECOG & $\geq 2$ vs. $0-1$ & 15.6371 & $<0.0001$ & 2.034 & 1.431 & 2.893 \\
\hline first-line treatment & $\begin{array}{l}\text { multidrug with DTIC vs. } \\
\text { mono DTIC } \\
\text { hormonal therapy vs. } \\
\text { mono DTIC }\end{array}$ & $\begin{array}{l}8.7797 \\
1.200\end{array}$ & $\begin{array}{l}0.0030 \\
0.1773\end{array}$ & $\begin{array}{l}1.349 \\
0.631\end{array}$ & $\begin{array}{l}1.107 \\
0.323\end{array}$ & $\begin{array}{l}1.644 \\
1.232\end{array}$ \\
\hline palliative resections & Y vs. N & 3.9347 & 0.0473 & 0.733 & 0.539 & 0.996 \\
\hline palliative radiotherapy & Y vs. N & 4.1804 & 0.0409 & 0.773 & 0.604 & 0.989 \\
\hline mestastasis during diagnosis & Y vs. N & 5.1636 & 0.0231 & 0.569 & 0.350 & 0.925 \\
\hline $\begin{array}{l}\text { metastasis from unknown } \\
\text { primary source }\end{array}$ & Y vs. N & 7.7595 & 0.0053 & 0.377 & 0.190 & 0.749 \\
\hline
\end{tabular}

were 3.5, 2.0, 1.8, respectively. A trend for shortening of the PFS after successive lines of systemic treatment is visible.

The PFS after successive lines of treatment is shown graphically in Figure 2. The PFS length after the fourth line may be thought-provoking, but because of the small size of the group it does not seem meaningful, or it is a group of patients with natural long course of disease.

\section{Multivariate analysis for OS}

The variables showing an independent influence on OS in the constructed model are shown in Table 5. Due to the scarcity of groups receiving respective types of therapy in the first line of treatment, it was decided to include only the largest groups in the multivariate analysis - those treated with dacarbazine in monotherapy or multidrug schemes with dacarbazine and hormonal therapy.

The variables independently influencing overall survival were: initial serum LDH level, initial serum aspartate transaminase (AspAT) level, initial performance status (ECOG), the type of first-line treatment used, women's age, metastases at the time of diagnosis, metastases from an unknown primary site, using palliative radiotherapy and palliative resections.

\section{Treatment toxicity}

The most common grade 3 and 4 adverse events were haematological disturbances (anaemia, thrombocytopenia, neutropenia), nausea and vomiting. Both haematological and non-haematological complications were found more commonly in multidrug chemotherapy with DTIC. In $9.5 \%$ of patients the complications resulted in premature treatment termination.

\section{Discussion}

Melanomas at the inoperable/metastatic stage are still incurable, and lead to death within a few months. In routine practice various kinds of therapy have been used, whose effectiveness is not always confirmed in clinical trials. The basic assessed parameter in the analysed large group of 437 patients was overall survival. The median OS in the whole analysed group was 7.1 months, with 1-, 2-, and 5-year survival rates of $32.3 \%, 12.5 \%$ and $3.9 \%$ respectively. Similar results were obtained by different authors who analysed overall survival of patients with inoperable, metastatic melanomas, with median OS ranging from 5.6 to 8.1 months [6, 7, 23, 2629]. In a meta-analysis of 83 research projects of stage II performed in the years 1974-1999 (6322 patients) [29] the OS median was 8.1 months, and 2- and 5 -year survival rates were $13.6 \%$ and $2.3 \%$ respectively. It is worth noting the significant differences between median OS before and after 1985: 5.8 and 8.9 months respectively. When taking confidence intervals for OS in the discussed analysis into consideration, the results of our group are fully within their range, which confirms the representativeness of the group.

A number of clinical factors which may affect OS in the patient group were analysed. In univariate analysis male sex proved to be a factor related to worse prognosis, which was not, however, confirmed in multivariate analysis. In some published studies male sex was a factor related to worse prognosis [6, 10, 26, 27, 32] (especially when assessing patients' survival in stages I-III), while in other works such a correlation was not confirmed [5, 13, 25, 28, 30, 31, 33-35]. We did not find an influence on OS by overall patient's age at the time of metastasis or by period of time between the melanoma diagnosis and metastases. However, we found a trend for worsening prognosis for younger patients and those whose metastasis has happened in a shorter time since the diagnosis. In the available literature, excluding a few works in which a positive influence of the time period before metastasis on OS was found [7, 35], this parameter was not statistically significant [5, 6, 10, 31-33]. Multivariate analysis also 
showed that the patients already diagnosed with metastasis have better prognosis (analysing only stage IV) than those patients whose metastasis occurred after removing the primary source or metastasis to lymph nodes. A similar correlation occurred between patients with metastasis from an unknown primary site and patients with established diagnosis of cutaneous melanoma. The fact is confirmed by other studies regarding outcomes of melanomas from an unknown primary site [36-39]. Another important factor is patient general status according to ZUBROD/ECOG/WHO scales at the time of therapy initiation. It has to be underlined that worsening patient's condition is connected with occupying vital organs, which results in worse prognosis for the group. In the present study we found, both in uni- and multivariate analysis, a statistically significant correlation between shortening of OS and decreasing patient's performance status (PS 0-1 vs. $\geq 2$ ) [5, 6, 10, 27, 28, 31-33]. In assessed biochemical tests a substantial influence on OS was shown in univariate analysis for LDH, AspAT and AIAT level in serum, and multi-factor analysis confirmed a relation between increased LDH and AspAT levels with shorten OS. Elevated lactate dehydrogenase (LDH) activity in serum is a negative prognostic factor, which was confirmed by several studies [5, 6, 23, 25, 26, 31]. In the TNM melanoma staging published by AJCC in 2001, elevated LDH level in serum was introduced to the stage IV melanoma classification, assigning patients to the category with the worst prognosis (IVc), which was the most important change in the metastatic melanoma categories in relation to the previous TNM staging [40]. However, the real biological meaning of elevated LDH activity in patients with advanced melanoma is still not fully understood. Another important factor affecting OS was the number of involved organs, which was not confirmed by multivariate analysis. The fact may be justified by the strong correlation between the number of occupied sites with bad prognosis and general condition, which often causes this variable to be excluded from multivariate analysis models. In uni- and multivariate analyses some authors have confirmed the influence of involved organs on the OS $[6,23,27,35]$. The localization of metastases is also an important prognostic factor. Data from the literature suggest that the most common locations of the first melanoma metastases are skin, subcutaneous tissues and lymph nodes $-59 \%$, lungs $-36 \%$, liver $-20 \%$, bones $-17 \%$, and brain $-20 \%$ [24]. The frequency of a particular location as the place of first metastasis was similar in the discussed material. In a series of studies it was demonstrated that visceral metastases are an independent factor which worsens the prognosis $[10,28,35]$. Similarly, in meta-analyses [40] which were basis for TNM staging, a considerable difference in OS between the group with involved nodes and soft tissues, and the group with occupied visceral organs and lungs was found. Also in the present study a statistical significance between particular sub-groups in stage IV was demonstrated. It is to be noted, however, that the differences in OS between particular groups are small. In the tested group the metastasis sites having a negative influence on OS in univariate analysis were lung and liver. This relationship was not confirmed in the multivariate analysis. In the literature, the most common negative factors for OS were metastases to the liver and/or brain
$[5,6,10,26-28,33,41,42]$. Some authors have also reported a negative influence of metastases in lungs [6, 26, 41], bones [6], skin and soft tissues [6]. Table 5 summarizes the most important studies analysing the influence of various factors on OS.

We have demonstrated, both in uni- and multivariate analyses, a statistically significant lengthening of OS in patients undergoing palliative resections. Performing palliative resections of metastases in carefully selected patients has an influence on OS documented in some studies, with the resections of single brain metastases especially positively impacting OS $[27,43,44]$. Also performing palliative radiotherapy has lengthened the OS in the analysed group. This relationship may be connected with the type of radiotherapy or with its wider use in curing brain metastases. In our study the influence of performed systemic treatment on the OS was demonstrated both in single- and multi-factor analyses. The relationship is worth underlining, as in already performed randomised clinical trials comparing different types of systemic treatment, none of these types of therapy showed significant benefits in terms of OS as compared to dacarbazine (DTIC) [11, 18, 20, 21, 45-50]. We have compared OS curves for all types of first-line treatment. In univariate analysis patients treated in the first-line with dacarbazine in monotherapy demonstrated the longest OS and PFS. In multivariate analysis a statistically significant longer OS in patients treated with DTIC in monotherapy was confirmed. However, it is worth noting that these results may be biased by several factors as no random choice of patients was used in any of the analysed groups, and the choice of the therapy depended on the doctor assigning patients to the treatment and on the current trends. The heterogeneity of the used drug regimens also must be stressed - CVD (cisplatin, vinblastine, DTIC), Dartmouth scheme (cisplatin, carmustine, DTIC, tamoxifen), biochemotherapy with interleukin 2 and/or interferon, and in single cases other drug combinations. The important fact is that multidrug therapy is used mostly in younger patients, those with higher stages, or those with visceral organ metastases, which may explain the better results of DTIC monotherapy. In already published randomized clinical trials and meta-analyses, no influence on OS was proven in patients treated with tamoxifen in monotherapy or in combinations with other drugs [47, 49-53]. Only one test showed a positive influence on OS of tamoxifen with dacarbazine over DTIC monotherapy [54]. Currently, using tamoxifen is not recommended in treating advanced metastatic melanoma patients. Dacarbazine has been invariably used in treating advanced melanomas since the 1970s, despite lack of randomized trials confirming its influence on OS. Despite such long use, dacarbazine has no definitely recommended standard dosage. The drug is used in a $220-250 \mathrm{mg} / \mathrm{m}^{2}$ dose for 4-5 days every 3-4 weeks, as well as in a $800-1000 \mathrm{mg} / \mathrm{m}^{2}$ dose every 3 weeks [11]. There are no objective studies comparing both schemes. One of the basic factors analysed in most clinical trials is assessment of the response to applied systemic treatment. It was believed that increasing the rate of objective responses would directly influence OS. According to our data such a relationship was observed in the whole group of patients, but it was not 
Table 5. Summary of research analysing prognostic factors in the group of patients with metastatic melanomas

\begin{tabular}{|c|c|c|c|c|c|}
\hline Research & $\begin{array}{c}\text { Time } \\
\text { of treatment }\end{array}$ & $\begin{array}{c}\text { Number } \\
\text { of patients }\end{array}$ & $\begin{array}{l}\text { OS - } \\
\text { median } \\
\text { (in months) }\end{array}$ & $\begin{array}{l}\text { PFS - } \\
\text { median } \\
\text { (in months) }\end{array}$ & Factors influencing OS negatively in multi-factor analyses \\
\hline Presant [33] & 1974-1978 & 277 & 6.0 & ne & $\begin{array}{l}\text { - male sex } \\
\text { - worse general condition } \\
\text { - occupied liver } \\
\text { - metastases in locations outside bone }\end{array}$ \\
\hline Balch [35] & 1955-1983 & 200 & 6.0 & ne & $\begin{array}{l}\text { - higher number of involved locations (organs) } \\
\text { - metastases in parenchymal organs } \\
\text { - shorter metastasis time }\end{array}$ \\
\hline Ryan [41] & 1975-1985 & 629 & 5.5 & ne & $\begin{array}{l}\text { - male sex } \\
\text { - worse general condition } \\
\text { - metastases in locations other than soft tissues and nodes } \\
\text { - higher number of involved (without bone) locations (organs) } \\
\text { - worse response to the treatment } \\
\text { - clinical symptoms (appetite loss, nausea/vomiting, no fever) }\end{array}$ \\
\hline Eton [23] & 1979-1989 & 318 & 8.0 & ne & $\begin{array}{l}\text { - male sex } \\
\text { - involvement of } \geq 2 \text { parenchymal organs } \\
\text { - elevated LDH level } \\
\text { - hypoalbuminemia }\end{array}$ \\
\hline Sirrot [26] & 1984-1991 & 284 & 7.4 & ne & $\begin{array}{l}\text { - male sex } \\
\text { - metastases in parenchymal organs } \\
\text { - elevated LDH level } \\
\text { - decreased albumin level } \\
\text { - thrombocytopenia }\end{array}$ \\
\hline Falkoson [42] & 1972-1992 & 236 & 6.3 & ne & $\begin{array}{l}\text { - worse general condition } \\
\text { - progression-free survival } \\
\text { - type of therapy (DTIC + IFN vs. DTIC, fotemustine vs. other drug) }\end{array}$ \\
\hline Barth [7] & $1971-1993$ & 1521 & 7.5 & ne & $\begin{array}{l}\text { - metastases in bones, liver, brain or lungs } \\
\text { - shorter time to metastasis } \\
\text { - primary illness stage }\end{array}$ \\
\hline
\end{tabular}

\begin{tabular}{|c|c|c|c|c|c|}
\hline Keiholz [55] & before 1994 & 681 & 10.5 & ne & $\begin{array}{l}\text { - worse general condition } \\
\text { - metastases location } \\
\text { - elevated LDH level }\end{array}$ \\
\hline Flaherty [28] & 1982-1995 & 813 & 5.6 & ne & $\begin{array}{l}\text { - worse general condition } \\
\text { - more than one involved location (organ) } \\
\text { - higher number of involved locations (organs) } \\
\text { - liver involvement } \\
\text { - time of metastasis }\end{array}$ \\
\hline
\end{tabular}

\begin{tabular}{|c|c|c|c|c|c|}
\hline Brand [44] & 1976-1996 & 442 & 7.0 & ne & $\begin{array}{l}\text { - worse general condition } \\
\text { - brain or skin metastases } \\
\text { - more than one involved location (organ) } \\
\text { - subsequent metastases } \\
\text { - palliative resections }\end{array}$ \\
\hline Manola [12] & 1975-1998 & 1362 & 6.4 & ne & $\begin{array}{l}\text { - male sex } \\
\text { - worse general condition } \\
\text { - more than one involved location (organ) } \\
\text { - metastases in parenchymal organs, lungs } \\
\text { - worse response to treatment } \\
\text { - precious immunotherapy (better prognosis) } \\
\text { - elevated LDH level } \\
\text { - elevated alkaline phosphatase level } \\
\text { - thrombocytopenia }\end{array}$ \\
\hline
\end{tabular}

\begin{tabular}{|c|c|c|c|c|c|}
\hline Korn [16] & 1975-2005 & 2100 & 6.2 & 1.7 & $\begin{array}{l}\text { - male sex } \\
\text { - worse general condition } \\
\text { - metastases in parenchymal organs } \\
\text { - metastases in brain }\end{array}$ \\
\hline $\begin{array}{l}\text { Minor }[50] \\
\text { (only biochemo- }\end{array}$ & $2002-2007$ & 135 & 16.6 & 7.6 & $\begin{array}{l}\text { - metastases in organs other than skin or lymph nodes } \\
\text { - elevated LDH level }\end{array}$ \\
\hline
\end{tabular}


confirmed in multivariate analysis. Similarly, it was not confirmed in several clinical studies, in which a statistically significant increase in objective responses did not influence OS $[21,22,46]$. Some authors have reported similar observations to us, that when a successive line of treatment is used, the objective response rate lowers. At present, the most important parameter influencing OS is progression-free survival (PFS) [10]. In the analysed group the median PFS during the first-line treatment was 3.5 months, which was similar to the data from the literature $[5,20]$. In our study the type of therapy was statistically significantly related to PFS and OS. It was also demonstrated that similar factors shorten PFS and OS. Those are: male gender, raised LDH, AIAT and AspAT levels, worse performance status, presence of liver metastases and higher stage according to the AJCC/UICC staging system. The correlation between the number of treatment lines and OS was also analysed. Longer OS in patients who received more lines of treatment might stem from survival bias, which means that the patient had to live until the next lines, and so by assumption lived longer. It is worth noting that in several patients, the treatment was changed after $2-3$ cycles due to progression or adverse events, which also affected the correlation between OS and number of treatment lines.

A trend for shorter PFS after successive lines of treatment was proven; median PFS for the first, second and third line of treatment was 3.5, 2.0, 1.8 months, respectively. The decreasing PFS times after successive lines of treatment make doubtful the utility of successive lines of cytotoxic chemotherapy in advanced melanoma.

To summarize, the analysed group comprises one of the largest ever analysed, contemporary groups of stage IV/unresectable stage III melanoma patients treated with systemic chemotherapy before the newer molecularly oriented treatments and anti-CTLA-4 immunotherapy. The results may provide a basis for proper selection of patients for clinical trials and treatment of some patients with classic chemotherapy in the era of modern drugs.

The authors declare no conflict of interest.

\section{References}

1. Didkowska J, Wojciechowska U, Tarkowski W, Zatoński W. Nowotwory złośliwe w Polsce w 2005 roku. Centrum Onkologii - Instytut 2007.

2. Ruka W, Krzakowski M, Placek W, et al. Czerniaki skóry - zasady postępowania diagnostyczno-terapeutycznego Onkol Prak Klin 2009; 5, 1: 20-32

3. Balch CM, Gershenwald JE, Soong S, et al. Final Version of 2009 AJCC Melanoma Staging and Classification J Clin Oncol 2009; 27: 6199-206.

4. Allen LE, Kupelnic B, Kumashiro D, et al. The combination of chemotherapy with II-2 and alpha IFN is more active than chemotherapy or immunotherapy alone in patients withmetastatic melanoma: a meta-analysis of 7711 patients with metastatic melanoma. Proc Am Soc Clin Oncol 1997; 16: 1781, p494a.

5. Eton O, Legha SS, Bedikian AY, et al. Sequential Biochemotherapy versus chemotherapy for metastatic melanoma: Results from a phase III randomised trials. J.Clin Oncol 2002; 20: 2045-52.

6. Manola J, Atkins M, Ibrahim J, Kirkwood J. Prognostis Factors in M: 3782-93.
7. Barth A, Wanek LA, Morton DL. Prognostic factors in 1521 melanoma patients with distant metastases. J Am Coll Surg 1995; 181: 193-201.

8. Lotze MT, Dallal RM, Kirkwood JM, et al. Cutaneous melanoma. in: Cancer principles \& Practice of Oncology. De Vita VT, Hellman S, Rosenberg SA (eds.). Lippincot Wiliams \& Wilkins. Philadelphia 2001, 2020.

9. Buzaid AC, Bedikian A, Houghton AN. Systemic chemotherapy and biochemotherapy, in Cutaneous melanoma. Balch CM, Houghton AN, Sober MD (eds.). Quality Medical Publishing, Inc. St. Louis, Missouri 1998, 406-18.

10. Korn EL, Liu PY, Lee SJ, et al. Meta-analysis of phase II cooperative group trials in metastatic stage IV melanoma to determine progression-free and overall survival benchmarks for future phase II trials. J Clin Oncol 2008; 26: 527-34.

11. Lui P., Cashin R., Machado M., et al. Treatments for metastatic melanoma: Synthesis of evidens from randomized trials. Cancer Treat Rev 2007; 33: 665-80.

12. Bhatia S., Tykodi S., Thompson J. Treatment of metastatic melanoma: Overview. Oncology 2009; 23960: 488-96.

13. Middleton MR, Grob JJ, Aaroson N, et al. Randomized phase III study of temozolomide versus dacarbazine in treatment of patients with advanced metastatic malignant melanoma. J Clin Oncol 2000;18:158-166.

14. Atkins MB, Kunkel L, Sznol M, Rosenberg SA. High-dose recombinant interleukin-2 therapy in patients with metastatic melanoma: long-term survival update. Cancer J Sci Am 2000; 6 Suppl 1: S11-4.

15. Atkins MB, Lotze MT, Dutcher JP, et al. High-dose recombinant interleukin 2 therapy for patients with metastatic melanoma: analysis of 270 patients treated between 1985 and 1993. J Clin Oncol 1999; 17: 2105-16.

16. Del Prete SA, Maurer LH, O'Donnell J, Forcier RJ, LeMarbre P. Combination chemotherapy with cisplatin, carmustine, dacarbazine, and tamoxifen in metastatic melanoma. Cancer Treat Rep. 1984 Nov;68(11):1403-5.

17. Lattanzi SC, Tosteson T, Chertoff J, et al. Dacarbazine, cisplatin and carmustine, with or without tamoxifen, for metastatic melanoma: 5-year follow-up. Melanoma Res 1995; 5: 365-9.

18. Chapman PB, Einhorn LH, Meyers ML, et al. Phase III multicenter randomized trial of the Dartmouth regimen versus dacarbazine in patients with metastatic melanoma. J Clin Oncol 1999; 17: 2745-51.

19. Legha SS, Ring S, Papadopoulos N, Plager C, Chawla S, Benjamin R. A prospective evaluation of a triple-drug regimen containing cisplatin, vinblastine, and dacarbazine (CVD) for metastatic melanoma. Cancer 1989; 64: 2024-9.

20. Bajetta E, Del Vecchio M, Nova P, et al. Multicenter phase III randomized trial of polychemotherapy (CVD regimen) versus the same chemotherapy (CT) plus subcutaneous interleukin-2 and interferon-alpha2b in metastatic melanoma. 2006; 17: 571-7.

21. Rutkowski P, Nowecki ZI, Dziewirski W, et al. Melanoma without a detectable primary site with metastases to lymph nodes. Dermatol Surg 2010; 36: 868-76.

22. Ives NJ, Stowe RL, Lorigan P, Wheatley K. Chemotherapy compared with biochemotherapy for the treatment of metastatic melanoma: a meta-analysis of 18 trials involving 2,621 patients. J Clin Oncol 2007; 25: 5426-34.

23. Eton O, Legha SS, Moon TE, et al. Prognostic factors for survival of Patients Treated Systemically for Disseminated melanoma. J Clin Oncol 1998; 16: 1103-1111

24. Czerniak zlośliwy. Skowronek J, Mackiewicz A, Żygulska-Mach H (ed.). Termedia, Poznań 1998.

25. Balch CM, Soong SJ, Gershenwakd JE, et al. Prognostic factors analisis of 17600 melanoma patients: Validation of the American Joint Committee on Cancer melanoma staging system. J Clin Oncol 2001; 19: 3622-34

26. Sirott MN, Bajorin DF, Wong GY, et al. Prognostic Factors in Patients with Metastatic Malignant melanoma. Cancer 1993; 72: 3091-8.

27. Brand CU, Ellwanger U, Stroebel W, et al. Prolonged survival of 2 years or longer for patients with disseminated melanoma. An analysis of related prognostic factors. Cancer 1997; 79: 2345-53. 
28. Unger JM, Flaherty LE, Liu PY, Albain KS, Sondak VK. Gender and other survival predictors in patients with metastatic melanoma on Southwest Oncology Group trials. Cancer 2001; 91: 1148-55.

29. Lee ML, Tomsu K, Von Eschen KB. Duration of survival for disseminated malignant melanoma: results of a meta-analysis. Melanoma Res 2000; 10: 81-92.

30. Keilholz U, Punt CJ, Gore M, et al. Dacarbazine, cisplatin, and interferon-alfa-2b with or without interleukin-2 in metastatic melanoma: a randomized phase III trial (18951) of the European Organisation for Research and Treatment of Cancer Melanoma Group. J Clin Oncol 2005; 23: 6747-55.

31. Minor DR, Moore D, Kim C, Kashani-Sabet M, Venna SS, Wang W, Boasberg P, O'Day S. Prognostic factors in metastatic melanoma patients treated with biochemotherapy and maintenance immunotherapy. Oncologist 2009; 14: 995-1002.

32. Ryan L, Kramar A, Borden E. Prognostic factors in metastatic melanoma. Cancer 1993; 71: 2995-3005.

33. Presant CA, Bartolucci AA. Prognostic factors in metastatic malignant melanoma: The Southeastern Cancer Study Group Experience. Cancer. 1982; 49: 2192-6.

34. Flaherty LE, Atkins M, Sosman J, et al. Outpatient biochemotherapy with interleukin-2 and interferon alfa- $2 b$ in patients with metastatic malignant melanoma: results of two phase II cytokine working group trials. J Clin Oncol 2001; 19: 3194-202.

35. Balch CM, Soong SJ, Murad TM, Smith JW, Maddox WA, Durant JR. A multifactorial analysis of melanoma. IV. Prognostic factors in 200 melanoma patients with distant metastases (stage III). J Clin Oncol 1983; 1: 126-34

36. Savoia P, Fava P, Osella-Abate S, Nardñ T, Comessatti A, Quaglino $P$, Bernengo MG.. Melanoma of unknown primary site: a 33 year experience at the Turin Melanoma Centre. Melanoma Res 2010; 20: 227-32.

37. Lee CC, Faries MB, Wanek LA, Morton DL. Improved survival for stage IV melanoma from an unknown primary site. J Clin Oncol 2009; 27: 3489-95.

38. Laveau F, Picot MC, Dereure O, Guilhou JJ, Guillot B. Metastatic melanoma of unknown primary site. Ann Dermatol Venereol 2001; 128: 893-8

39. Vijuk G, Coates AS. Survival of patients with visceral metastatic melanoma from an occult primary lesion: a retrospective matched cohort study. Ann Oncol 1998; 9: 419-22.

40. Balch CM, Buzaid AC, Soong SJ, et al. Final version of the American Joint Committee on Cancer staging system for cutaneous melanoma. J Clin Oncol 2001; 19: 3635-48.

41. Ryan L, Kramar A., Borden E. Prognostic factors in Metastatic melanoma. Cancer 1993; 71: 2995-3005

42. Falkson Cl, Falkson HC. Prognostic factors in metastatic malignant melanoma. An analysis of 236 patients treated on clinical research studies at the Department of Medical Oncology, University of Pretoria, South Africa from 1972-1992. Oncology 1998; 55: 59-64.

43. Essner R. Surgical treatment of malignant melanoma. Surg Clin North Am 2003; 83: 109-56.

44. Young SE, Martinez SR, Essner R. The role of surgery in treatment of stage IV melanoma. J Surg Oncol 2006; 94: 344-51.

45. Thomson DB, Adena M, McLeod GR, et al. Interferon-alpha 2a does not improve response or survival when combined with dacarbazine in metastatic malignant melanoma: results of a multi-institutional Australian randomized trial. Melanoma Res 1993; 3: 133-8.

\section{Address for correspondence}

Bożena Cybulska-Stopa

Klinika Nowotworów Układowych i Uogólnionych

Centrum Onkologii Oddział w Krakowie

Garncarska 11

31-115 Kraków

e-mail: bcybulskastopa@vp.pl

Submitted: 11.04 .2012

Accepted: $\quad 17.07 .2012$ 